PHYSICAL REVIEW D 96, 051103(R) (2017)

\title{
Observation of charmless baryonic decays $B_{(s)}^{0} \rightarrow p \bar{p} h^{+} h^{\prime-}$
}

\author{
R. Aaij et al. \\ (LHCb Collaboration) \\ (Received 28 April 2017; published 27 September 2017)
}

\begin{abstract}
Decays of $B^{0}$ and $B_{s}^{0}$ mesons to the charmless baryonic final states $p \bar{p} h^{+} h^{\prime-}$, where $h$ and $h^{\prime}$ each denote a kaon or a pion, are searched for using the LHCb detector. The analysis is based on a sample of protonproton collision data collected at center-of-mass energies of 7 and $8 \mathrm{TeV}$, corresponding to an integrated luminosity of $3 \mathrm{fb}^{-1}$. Four-body charmless baryonic $B_{s}^{0}$ decays are observed for the first time. The decays $B_{s}^{0} \rightarrow p \bar{p} K^{+} K^{-}, B_{s}^{0} \rightarrow p \bar{p} K^{ \pm} \pi^{\mp}, B^{0} \rightarrow p \bar{p} K^{ \pm} \pi^{\mp}$ and $B^{0} \rightarrow p \bar{p} \pi^{+} \pi^{-}$are observed with a significance greater than 5 standard deviations; evidence at 4.1 standard deviations is found for the $B^{0} \rightarrow p \bar{p} K^{+} K^{-}$ decay and an upper limit is set on the branching fraction for $B_{s}^{0} \rightarrow p \bar{p} \pi^{+} \pi^{-}$. Branching fractions in the kinematic region $m(p \bar{p})<2850 \mathrm{MeV} / c^{2}$ are measured relative to the $B^{0} \rightarrow J / \psi(\rightarrow p \bar{p}) K^{*}(892)^{0}$ channel.
\end{abstract}

DOI: 10.1103/PhysRevD.96.051103

In recent years, studies by the $\mathrm{LHCb}$ Collaboration have greatly increased the knowledge of the decays of $B$ mesons to final states containing baryons. The first observation of a baryonic $B_{c}^{+}$decay was reported in 2014 [1], and $\mathrm{LHCb}$ recently reported the first observation of a baryonic $B_{s}^{0}$ decay [2], the last of the four $B$ meson species for which a baryonic decay mode had yet to be observed.

Primary areas of interest in baryonic $B$ decays include the hierarchy of branching fractions to the various decay modes, the presence of resonances and the existence of a threshold enhancement in the baryon-antibaryon mass spectrum $[3,4]$. The first evidence of $C P$ violation in baryonic $B$ decays has been reported from an analysis of $B^{+} \rightarrow p \bar{p} K^{+}$decays [5]. It is of great interest to search for further manifestations of $C P$ violation in baryonic $B$ decays, e.g. with so-called triple-product correlations (TPCs); see Ref. [6] and references therein. For certain decays, asymmetries of up to $20 \%$ are predicted [7]. Four-body decays are particularly suited for this approach since the definitions of the TPCs do not involve the spins of the final-state particles, unlike the TPCs in three-body decays $[6,8]$.

This paper presents a search for the decays of $B^{0}$ and $B_{s}^{0}$ mesons to the four-body charmless baryonic final states $p \bar{p} h^{+} h^{\prime-}$, where $h$ and $h^{\prime}$ each denote a kaon or a pion. The inclusion of charge-conjugate processes is implied, unless otherwise indicated. For simplicity, the charges of the $h^{+} h^{\prime-}$ combinations will be omitted unless necessary. The branching fractions of these baryonic decays are measured relative to the $B^{0} \rightarrow J / \psi(\rightarrow p \bar{p}) K^{*}(892)^{0}$ channel. So far only the

*Full author list given at the end of the article.

Published by the American Physical Society under the terms of the Creative Commons Attribution 4.0 International license. Further distribution of this work must maintain attribution to the author(s) and the published article's title, journal citation, and DOI. resonant decay $B^{0} \rightarrow p \bar{p} K^{*}(892)^{0}$ has been seen by the $B A B A R$ [9] and Belle [10] collaborations, which measured its branching fraction to be $\mathcal{B}\left(B^{0} \rightarrow p \bar{p} K^{*}(892)^{0}\right)=$ $\left(1.24_{-0.25}^{+0.28}\right) \times 10^{-6}[11]$. An upper limit $\mathcal{B}\left(B^{0} \rightarrow p \bar{p} \pi^{+} \pi^{-}\right)<$ $2.5 \times 10^{-4}$ at $90 \%$ confidence level has been set by the CLEO Collaboration [12].

The data sample analyzed corresponds to an integrated luminosity of $1 \mathrm{fb}^{-1}$ of proton-proton collision data at a center-of-mass energy of $7 \mathrm{TeV}$ and $2 \mathrm{fb}^{-1}$ at $8 \mathrm{TeV}$. The $\mathrm{LHCb}$ detector $[13,14]$ is a single-arm forward spectrometer covering the pseudorapidity range $2<\eta<5$, designed for the study of particles containing $b$ or $c$ quarks. The detector elements that are particularly relevant to this analysis are as follows: a silicon-strip vertex detector surrounding the proton-proton interaction region that allows $c$ and $b$ hadrons to be identified from their characteristically long flight distance; a tracking system that provides a measurement of momentum, $p$, of charged particles; two ring-imaging Cherenkov detectors that are able to discriminate between different species of charged hadrons; and calorimeter and muon systems for the measurement of photons and neutral hadrons, and the detection of penetrating charged particles. Simulated data samples, produced with software described in Refs. [15-20], are used to evaluate the response of the detector and to investigate possible sources of background.

Real-time event selection is performed by a trigger [21] that consists of a hardware stage, based on information from the calorimeter and muon systems, followed by a software stage, which performs a full event reconstruction. The hardware trigger stage requires events to have a muon with high transverse momentum, $p_{\mathrm{T}}$, or a hadron, photon or electron with high transverse energy in the calorimeters. Signal candidates may come from events where the hardware trigger was caused either by signal particles or by other particles in the event. The software trigger requires a two-, three- or four-track secondary vertex with a 
significant displacement from any primary proton-proton interaction vertices (PVs). At least one charged particle must have $p_{\mathrm{T}}>1.6 \mathrm{GeV} / c$ and be inconsistent with originating from a PV. A multivariate algorithm [22] is used for the identification of secondary vertices consistent with the decay of a $b$ hadron.

The final selection of $B_{(s)}^{0}$ candidates, formed by combining four charged hadron candidates-a proton, an antiproton and an oppositely charged pair of light mesons-is carried out with a filtering stage, followed by requirements on the response of a boosted decision tree (BDT) classifier [23,24] and on particle identification (PID). The filtering stage includes requirements on the quality, $p, p_{\mathrm{T}}$ and $\chi_{\mathrm{IP}}^{2}$ of the tracks, loose PID requirements and an upper limit on the $p \bar{p}$ invariant mass; the $\chi_{\mathrm{IP}}^{2}$ is defined as the difference between the vertex-fit $\chi^{2}$ of a PV reconstructed with and without the track in question. Each $B_{(s)}^{0}$ candidate must have a good-quality vertex that is displaced from the associated PV (that with which it forms the smallest $\chi_{\mathrm{IP}}^{2}$ ), must satisfy $p$ and $p_{\mathrm{T}}$ requirements, and must have a reconstructed invariant mass close to that of a $B_{(s)}^{0}$ meson under the signal mass hypothesis. A requirement is also imposed on the angle $\vartheta_{\text {dir }}$ between the candidate momentum vector and the line between the associated PV and the candidate decay vertex.

There are 15 input quantities to the BDT classifier: $p_{\mathrm{T}}, \eta$, $\chi_{\mathrm{IP}}^{2}, \vartheta_{\text {dir }}$ and the flight distance of the $B_{(s)}^{0}$ candidate; the quality of the $B_{(s)}^{0}$ vertex fit; the $p_{\mathrm{T}}$ and $\chi_{\mathrm{IP}}^{2}$ of the tracks; and the largest distance of closest approach between any pair of tracks. The BDT is trained using simulated $B_{(s)}^{0} \rightarrow$ $p \bar{p} h h^{\prime}$ signal candidates, generated with uniform distributions over phase space, and events in a high sideband of the $p \bar{p} K \pi$ invariant mass in data $[m(p \bar{p} K \pi)$ in the range $5450-5550 \mathrm{MeV} / c^{2}$ ] to represent the background. Tight PID requirements are applied to all final-state particles to reduce the combinatorial background, suppress the crossfeed backgrounds between the different $p \bar{p} h h^{\prime}$ final states-background from other signal decays where one particle is misidentified - and ensure that the data sets for the three $p \bar{p} h h^{\prime}$ final states are mutually exclusive. For each final state individually, the requirements on the PID and BDT response are optimized for the signal significance using simulation samples for the signal. After all selection requirements are applied, approximately $3 \%$ of events with at least one candidate also contain a second candidate; a candidate is then selected at random. The efficiency of the full reconstruction and selection, including the acceptance and the trigger selection, is approximately $0.1 \%$.

To reject contributions from intermediate charm states, candidates with $h h^{\prime}$ invariant mass consistent with a $D^{0}$ meson or $p h h^{\prime}$ invariant mass consistent with a $\Lambda_{c}^{+}$baryon are removed. The contribution from the charmonium region is removed by requiring the invariant mass of the $p \bar{p}$ pair to be less than $2850 \mathrm{MeV} / \mathrm{c}^{2}$, similar to the procedure in Refs. [5,25]. This last requirement is not applied to the normalization mode $B^{0} \rightarrow J / \psi K^{*}(892)^{0}$, where the vector mesons are reconstructed in the $J / \psi \rightarrow p \bar{p}$ and $K^{*}(892)^{0} \rightarrow K^{+} \pi^{-}$decay modes. All the other steps of the selection for the signal and the normalization modes are shared in common.

The yields of the signal decays are obtained from a simultaneous unbinned extended maximum likelihood fit to the $B_{(s)}^{0}$ candidate invariant mass distributions in the three $p \bar{p} h h^{\prime}$ final states in the range $5165-5525 \mathrm{MeV} / c^{2}$. This approach accounts for potential cross feed from one channel to another due to particle misidentification. Each signal component is modeled with a double-sided Crystal Ball (DSCB) function [26]. For each signal the tail parameters of the DSCB functions are determined from simulation. The peak position of the $B^{0}$ signals is common to the three final states, while the difference between the peak positions of the $B^{0}$ and $B_{s}^{0}$ signals is constrained to its known value [11]. The width of the $B^{0}$ signal is a free parameter in the $p \bar{p} K \pi$ final state and it is related to the width in the other two final states by scale factors determined from simulation. The same applies to the width of the $B_{s}^{0}$ signals, which is a free parameter only in the $p \bar{p} K K$ final state.

For each final state the dominant $B_{(s)}^{0} \rightarrow p \bar{p} h h^{\prime}$ crossfeed background is included: the $B^{0} \rightarrow p \bar{p} K \pi$ mode in the $p \bar{p} K K$ and $p \bar{p} \pi \pi$ invariant mass distributions, and the $B^{0} \rightarrow p \bar{p} \pi \pi$ mode in the $p \bar{p} K \pi$ spectrum. Each cross-feed background is modeled with a DSCB function with all the shape parameters fixed according to simulation; the yield is fixed relative to the yield in the correctly reconstructed final state taking into account the (mis)identification probabilities calibrated using data, as described below. In addition, a combinatorial background component modeled by an exponential function, with both parameters free to vary, is present for each final state.

The yield of the normalization decay is determined from a separate simultaneous fit to the $p \bar{p} K \pi, p \bar{p}$ and $K \pi$ invariant mass distributions in the ranges $5180-5380 \mathrm{MeV} / c^{2}$, $3047-3147 \mathrm{MeV} / c^{2}$ and $642-1092 \mathrm{MeV} / c^{2}$, respectively. The $B^{0} \rightarrow J / \psi K^{*}(892)^{0}$ component is parametrized in the $K \pi$ invariant mass distribution by a relativistic spin-1 BreitWigner function and in the $p \bar{p} K \pi$ and $p \bar{p}$ invariant mass distributions by DSCB functions with the tail parameters fixed from simulation. The $K \pi \mathrm{S}$-wave component is modeled in the $K \pi$ invariant mass distribution by the LASS parametrization $[27,28]$ that describes nonresonant and $K_{0}^{*}(1430)^{0} \mathrm{~S}$-wave contributions; this component is modeled in the $p \bar{p} K \pi$ and $p \bar{p}$ invariant mass distributions with the same shape as the $B^{0} \rightarrow J / \psi K^{*}(892)^{0}$ component. A combinatorial background component modeled by a freely varying exponential function is also present in each spectrum. 

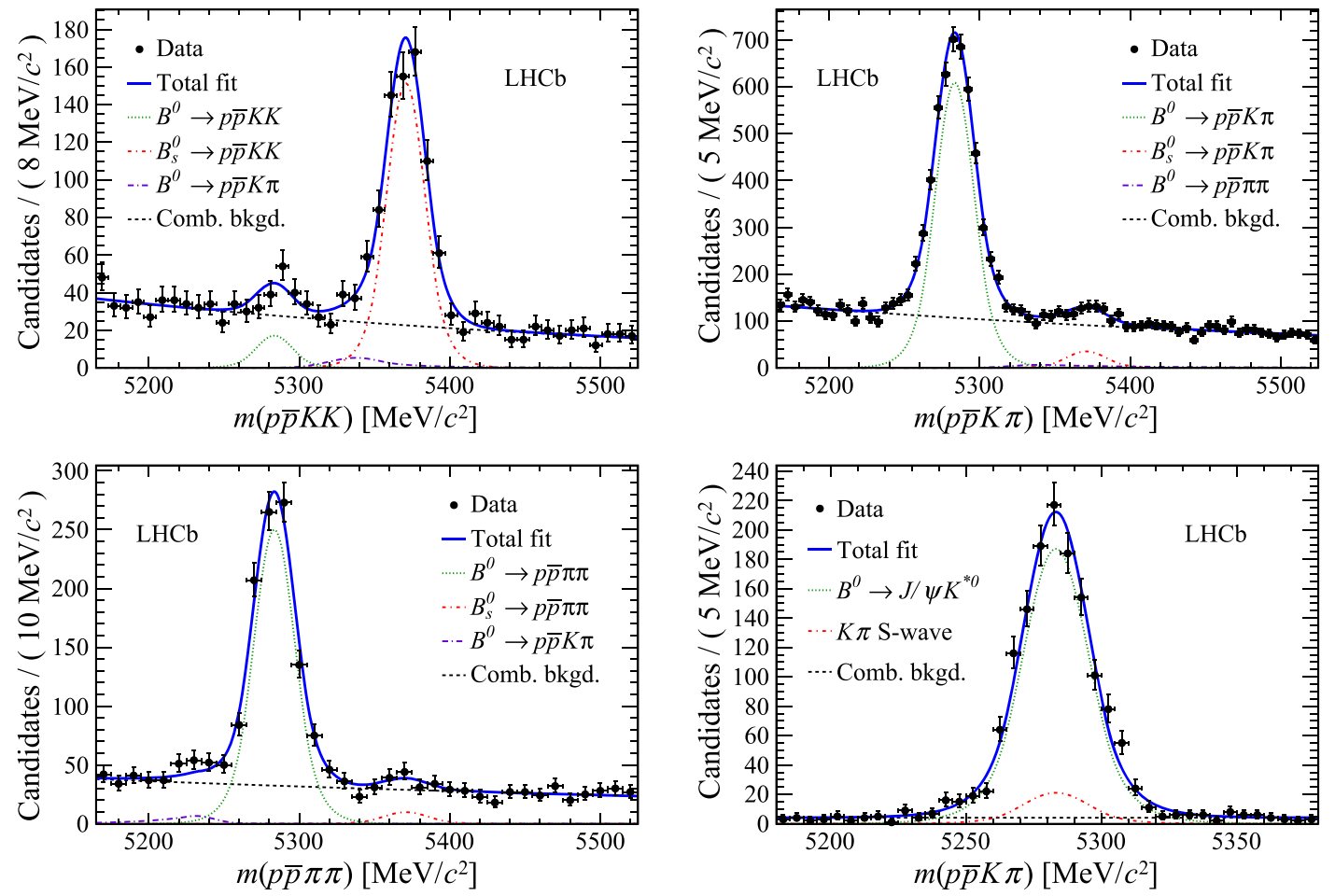

FIG. 1. Invariant mass distributions for $B_{(s)}^{0}$ candidates in the (top left) $p \bar{p} K K$, (top right) $p \bar{p} K \pi$, (bottom left) $p \bar{p} \pi \pi$ final state and (bottom right) invariant mass distribution of $B^{0} \rightarrow J / \psi K^{*}(892)^{0}$ in the $p \bar{p} K \pi$ final state. The results of the fits are shown with blue solid lines. In the first three figures signals for $B^{0}$ and $B_{s}^{0}$ decays are shown, respectively, with green dotted and red dotted-dashed lines, combinatorial backgrounds are shown with black dashed lines and cross-feed backgrounds are shown with violet dotted-dashed lines. In the bottom right figure the normalization signal is shown with a green dotted line, the $K \pi \mathrm{S}$-wave component is displayed with a red dotted-dashed line and the combinatorial background with a black dashed line.

The $p \bar{p} h h^{\prime}$ invariant mass distributions with the results of the fit overlaid are shown in Fig. 1 while the signal yields and the significances are collected in Table I. The significance of each of the signal modes is determined from the change in likelihood when the corresponding yield is fixed to zero, with systematic uncertainties taken into account [29]. The $B_{s}^{0} \rightarrow p \bar{p} K \pi, B^{0} \rightarrow p \bar{p} K K$ and $B_{s}^{0} \rightarrow p \bar{p} \pi \pi$ modes are found to have significances of 6.5 standard deviations $(\sigma), 4.1 \sigma$ and $2.6 \sigma$, respectively, while the other signal modes have significances greater than $25 \sigma$.

The branching fractions of the $B_{(s)}^{0} \rightarrow p \bar{p} h h^{\prime}$ decays are determined relative to the visible branching fraction of the $B^{0} \rightarrow J / \psi K^{*}(892)^{0}$ decay using

$$
\begin{aligned}
& \frac{\mathcal{B}\left(B_{(s)}^{0} \rightarrow p \bar{p} h h^{\prime}\right)}{\mathcal{B}_{\text {vis }}\left(B^{0} \rightarrow J / \psi K^{*}(892)^{0}\right)} \\
= & \frac{\mathcal{N}^{\operatorname{corr}}\left(B_{(s)}^{0} \rightarrow p \bar{p} h h^{\prime}\right)}{\mathcal{N}^{\operatorname{corr}}\left(B^{0} \rightarrow J / \psi K^{*}(892)^{0}\right)}\left(\times \frac{f_{d}}{f_{s}}\right),
\end{aligned}
$$

where $f_{s} / f_{d}=0.259 \pm 0.015$ (included only for the $B_{s}^{0}$ ) is the ratio of $b$ hadronization probabilities, $f_{q}$, to the hadron $B_{q}$ [30], and $\mathcal{N}^{\text {corr }}$ denotes efficiency-corrected fitted signal yields. The yields are obtained from the mass fits, while simulation is used to evaluate the contribution to the efficiency from each stage of the selection except for the effect of the PID criteria. The latter is determined from calibration data samples of kinematically identified pions, kaons and protons originating from the decays $D^{*+} \rightarrow D^{0}\left(\rightarrow K^{-} \pi^{+}\right) \pi^{+}, \Lambda \rightarrow p \pi^{-}$and $\Lambda_{c}^{+} \rightarrow p K^{-} \pi^{+}$and weighted according to the kinematics of the signal particles $[31,32]$. For each final state the efficiencies are determined as a function of the position in phase space, and efficiency corrections for each candidate are applied using the method of Ref. [33] to take the variation over the phase space into account. Explicitly, $\mathcal{N}^{\text {corr }}=\sum_{i} \mathcal{W}_{i} / \varepsilon_{i}$, where the sum runs over the candidates in the fit, $\mathcal{W}_{i}$ is the sWeight for candidate $i$ determined with the sPlot method [34] and $\varepsilon_{i}$ is the efficiency for the candidate $i$ which depends only on its position in the five-dimensional phase space. The visible branching fraction of the normalization mode, defined as $\mathcal{B}\left(B^{0} \rightarrow J / \psi K^{*}(892)^{0}\right) \times \mathcal{B}(J / \psi \rightarrow p \bar{p}) \times$ $\mathcal{B}\left(K^{*}(892)^{0} \rightarrow K^{+} \pi^{-}\right), \quad$ is $\quad \mathcal{B}_{\text {vis }}\left(B^{0} \rightarrow J / \psi K^{*}(892)^{0}\right)=$ $(1.68 \pm 0.12) \times 10^{-6}$, where the $B^{0} \rightarrow J / \psi K^{*}(892)^{0}$ branching fraction is taken from Ref. [35] and the others from Ref. [11].

The branching fraction of each signal mode is reported in Table I. The significance for the $B_{s}^{0} \rightarrow p \bar{p} \pi \pi$ mode is less than $3 \sigma$; an upper limit on its branching fraction is found to be 
TABLE I. Fitted yields, signal yield significances and branching fractions computed using Eq. (1). The uncertainties on the yields are statistical only. The first uncertainty on each branching fraction is statistical; the second is systematic; the third comes from the uncertainty on the branching fraction of the normalization mode; and the fourth, where present, is due to the uncertainty on $f_{d} / f_{s}$.

\begin{tabular}{|c|c|c|c|}
\hline Decay channel & Yield $\mathcal{N}$ & Significance $(\sigma)$ & Branching fraction $/ 10^{-6}$ \\
\hline$B^{0} \rightarrow p \bar{p} K K$ & $68 \pm 17$ & 4.1 & $0.113 \pm 0.028 \pm 0.011 \pm 0.008$ \\
\hline$B^{0} \rightarrow p \bar{p} K \pi$ & $4155 \pm 83$ & $>25$ & $5.9 \pm 0.3 \pm 0.3 \pm 0.4$ \\
\hline$B^{0} \rightarrow p \bar{p} \pi \pi$ & $902 \pm 35$ & $>25$ & $2.7 \pm 0.1 \pm 0.1 \pm 0.2$ \\
\hline$B_{s}^{0} \rightarrow p \bar{p} K K$ & $635 \pm 32$ & $>25$ & $4.2 \pm 0.3 \pm 0.2 \pm 0.3 \pm 0.2$ \\
\hline$B_{s}^{0} \rightarrow p \bar{p} K \pi$ & $246 \pm 39$ & 6.5 & $1.30 \pm 0.21 \pm 0.11 \pm 0.09 \pm 0.08$ \\
\hline$B_{s}^{0} \rightarrow p \bar{p} \pi \pi$ & $39 \pm 16$ & 2.6 & $0.41 \pm 0.17 \pm 0.04 \pm 0.03 \pm 0.02$ \\
\hline$B^{0} \rightarrow J / \psi K^{*}(892)^{0}$ & $1216 \pm 45$ & - & - \\
\hline
\end{tabular}

$$
\mathcal{B}\left(B_{s}^{0} \rightarrow p \bar{p} \pi \pi\right)<6.6 \times 10^{-7} \text { at } 90 \% \text { confidence level, }
$$

by integrating the likelihood after multiplying by a prior probability distribution that is uniform in the region of a positive branching fraction. The values of the ratios of branching fractions between different $B_{(s)}^{0} \rightarrow p \bar{p} h h^{\prime}$ decay modes are reported in Table II.

The signal distributions in $m\left(h h^{\prime}\right)$ and $m(p \bar{p})$ are obtained by subtracting the background using the sPlot technique [34], with the $B_{(s)}^{0}$ candidate invariant mass as the discriminating variable. Per-candidate weights are applied to correct for the variation of the selection efficiency over the phase space. Figure 2 shows the $h h^{\prime}$ invariant mass distributions of the $B^{0} \rightarrow p \bar{p} K \pi, B_{s}^{0} \rightarrow p \bar{p} K K$ and $B^{0} \rightarrow$ $p \bar{p} \pi \pi$ decay modes. A peak from a vector meson is identifiable in each mass spectrum, corresponding to a $K^{*}(892)^{0}$, a $\phi(1020)$ and a $\rho(770)^{0}$ meson, respectively. The $p \bar{p}$ invariant mass distributions are also shown for the same decay modes. An enhancement near threshold, typical in baryonic $B$ decays [3,4], is clearly visible in each case. Detailed amplitude analyses of the $B_{(s)}^{0} \rightarrow p \bar{p} h h^{\prime}$ decays will be of interest with larger samples.

The sources of systematic uncertainty on the absolute branching fractions and on the ratios of branching fractions arise from the fit model; the knowledge of the efficiencies; and, where appropriate, from the uncertainties on the branching fraction of the normalization mode and on the ratio of $b$-quark hadronization probabilities. Pseudoexperiments are used to estimate the effect of using alternative shapes for the fit components, or of including

TABLE II. Ratios of branching fractions among different $B_{(s)}^{0} \rightarrow p \bar{p} h h^{\prime}$ modes. The first uncertainty is statistical; the second is systematic; and the third, where present, comes from the uncertainty on $f_{d} / f_{s}$.

\begin{tabular}{lc}
\hline \hline $\mathcal{B}\left(B^{0} \rightarrow p \bar{p} K K\right) / \mathcal{B}\left(B^{0} \rightarrow p \bar{p} K \pi\right)$ & $0.019 \pm 0.005 \pm 0.002$ \\
$\mathcal{B}\left(B^{0} \rightarrow p \bar{p} \pi \pi\right) / \mathcal{B}\left(B^{0} \rightarrow p \bar{p} K \pi\right)$ & $0.46 \pm 0.02 \pm 0.02$ \\
$\mathcal{B}\left(B_{s}^{0} \rightarrow p \bar{p} K \pi\right) / \mathcal{B}\left(B^{0} \rightarrow p \bar{p} K \pi\right)$ & $0.22 \pm 0.04 \pm 0.02 \pm 0.01$ \\
$\mathcal{B}\left(B_{s}^{0} \rightarrow p \bar{p} K \pi\right) / \mathcal{B}\left(B_{s}^{0} \rightarrow p \bar{p} K K\right)$ & $0.31 \pm 0.05 \pm 0.02$ \\
\hline \hline
\end{tabular}

additional components in the fit. In particular, the effect of adding other cross-feed backgrounds, partially reconstructed backgrounds and components coming from $\Lambda_{b}^{0}$ decays have been investigated. These are the dominant sources of systematic uncertainty for the $B^{0} \rightarrow p \bar{p} K K$ and $B_{s}^{0} \rightarrow p \bar{p} \pi \pi$ modes. The effect of fixing the yields of the cross-feed backgrounds based on the (mis)identification probabilities is also assessed by varying these probabilities within their uncertainties. Intrinsic biases in the fitted yields are investigated with pseudoexperiments and are found to be negligible. Uncertainties on the efficiencies arise due to the limited size of the simulation samples, the uncertainty on their evaluated distributions across the phase space of the decays and from possible residual differences between data and simulation. The unknown decay kinematics are the principal source of systematic uncertainty for the $B_{s}^{0} \rightarrow$ $p \bar{p} K \pi$ mode, while for the $B_{s}^{0} \rightarrow p \bar{p} K K, B^{0} \rightarrow p \bar{p} K \pi$ and $B^{0} \rightarrow p \bar{p} \pi \pi$ modes the dominant source of systematic uncertainty comes from the uncertainty on the efficiency of the hardware stage of the trigger. As the efficiencies depend on the signal decay-time distribution, the effect coming from the different lifetimes of the $B_{s}^{0}$ mass eigenstates has been evaluated. The systematic uncertainties due to the vetoes of charm hadrons are also included.

In summary, a search for the four-body charmless baryonic decays $B_{(s)}^{0} \rightarrow p \bar{p} h h^{\prime}$ has been carried out by the LHCb Collaboration with a sample of proton-proton collision data corresponding to an integrated luminosity of $3 \mathrm{fb}^{-1}$. First observations are obtained for the decays $B^{0} \rightarrow p \bar{p} \pi \pi, \quad$ nonresonant $\quad B^{0} \rightarrow p \bar{p} K \pi, \quad B_{s}^{0} \rightarrow p \bar{p} K K$ and $B_{s}^{0} \rightarrow p \bar{p} K \pi$, while first evidence is reported for the $B^{0} \rightarrow p \bar{p} K K$ mode and an upper limit is set on the $B_{s}^{0} \rightarrow p \bar{p} \pi \pi$ branching fraction. In particular, four-body baryonic $B_{s}^{0}$ decays are observed for the first time and a threshold enhancement in the baryon-antibaryon mass spectra is confirmed for baryonic $B_{s}^{0}$ decays [2].

The LHCb Collaboration has recently published studies of $C P$ violation with four-body $\Lambda_{b}^{0} \rightarrow p h^{-} h^{+} h^{-}$decays studying triple-product correlations, and presented first evidence for $C P$ violation in baryons [36]. The decays of $B^{0}$ and $B_{s}^{0}$ mesons to $p \bar{p} h h^{\prime}$ final states reported in this 

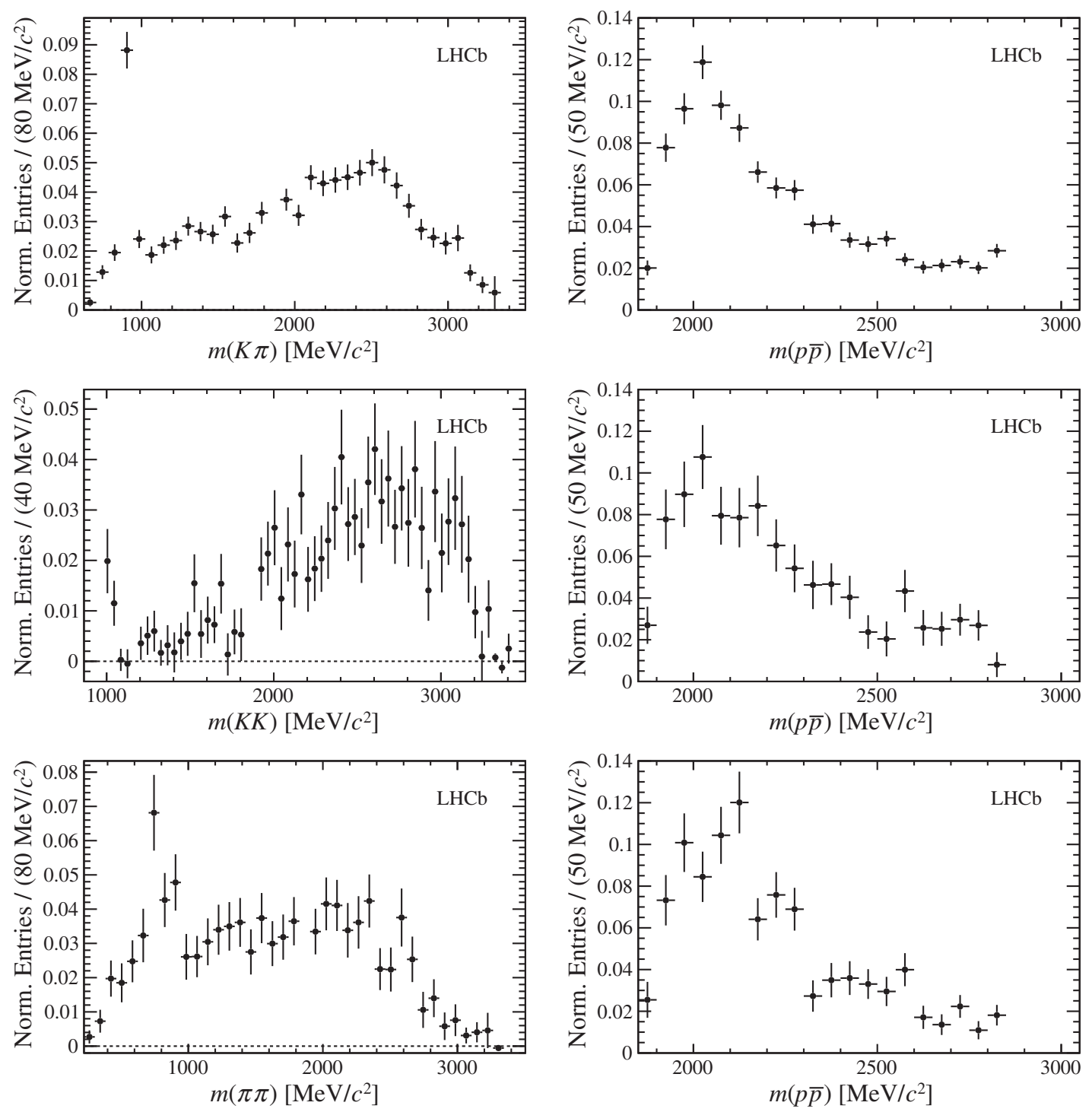

FIG. 2. Efficiency-corrected and background-subtracted (left) $m\left(h h^{\prime}\right)$ and (right) $m(p \bar{p})$ distributions from (top) $B^{0} \rightarrow p \bar{p} K \pi$, (middle) $B_{s}^{0} \rightarrow p \bar{p} K K$, and (bottom) $B^{0} \rightarrow p \bar{p} \pi \pi$ candidates. Events with entries in the charmonium or $D^{0}$ mass regions have been removed from the samples. All distributions are normalized to unity.

paper may be used in the future for similar studies of $C P$ violation in baryonic $B$ decays.

\section{ACKNOWLEDGMENTS}

We express our gratitude to our colleagues in the CERN accelerator departments for the excellent performance of the LHC. We thank the technical and administrative staff at the LHCb institutes. We acknowledge support from CERN and from the national agencies: CAPES, CNPq, FAPERJ and FINEP (Brazil); MOST and NSFC (China); CNRS/IN2P3 (France); BMBF, DFG and MPG (Germany); INFN (Italy); NWO (The Netherlands); MNiSW and NCN (Poland); MEN/IFA (Romania); MinES and FASO (Russia); MinECo (Spain); SNSF and SER (Switzerland); NASU (Ukraine); STFC (United Kingdom); NSF (USA).
We acknowledge the computing resources that are provided by CERN, IN2P3 (France), KIT and DESY (Germany), INFN (Italy), SURF (The Netherlands), PIC (Spain), GridPP (United Kingdom), RRCKI and Yandex LLC (Russia), CSCS (Switzerland), IFIN-HH (Romania), CBPF (Brazil), PL-GRID (Poland) and OSC (USA). We are indebted to the communities behind the multiple open source software packages on which we depend. Individual groups or members have received support from the $\mathrm{AvH}$ Foundation (Germany); EPLANET, Marie SkłodowskaCurie Actions and ERC (European Union); Conseil Général de Haute-Savoie, Labex ENIGMASS and OCEVU, Région Auvergne (France); RFBR and Yandex LLC (Russia); GVA, XuntaGal and GENCAT (Spain); Herchel Smith Fund, The Royal Society, Royal Commission for the Exhibition of 1851 and the Leverhulme Trust (United Kingdom). 
[1] R. Aaij et al. (LHCb Collaboration), First Observation of a Baryonic $B_{c}^{+}$Decay, Phys. Rev. Lett. 113, 152003 (2014).

[2] R. Aaij et al. (LHCb Collaboration), First Observation of a Baryonic $B_{s}^{0}$ Decay, Phys. Rev. Lett. 119, 041802 (2017).

[3] W.-S. Hou and A. Soni, Pathways to Rare Baryonic B Decays, Phys. Rev. Lett. 86, 4247 (2001).

[4] A. J. Bevan et al. (BABAR and Belle Collaborations), The physics of the B factories, Eur. Phys. J. C 74, 3026 (2014).

[5] R. Aaij et al. (LHCb Collaboration), Evidence for $C P$ Violation in $B^{+} \rightarrow p \bar{p} K^{+}$Decays, Phys. Rev. Lett. 113, 141801 (2014).

[6] C. Q. Geng and Y. K. Hsiao, Direct $C P$ and T violation in baryonic B decays, Int. J. Mod. Phys. A A23, 3290 (2008).

[7] C. Q. Geng, Y. K. Hsiao, and J. N. Ng, Direct CP Violation in $B^{ \pm} \rightarrow p \bar{p} K^{(*) \pm}$, Phys. Rev. Lett. 98, 011801 (2007).

[8] M. Gronau and J. L. Rosner, Triple product asymmetries in $K, D_{(s)}$ and $B_{(s)}$ decays, Phys. Rev. D 84, 096013 (2011).

[9] B. Aubert et al. (BABAR Collaboration), Evidence for the $B^{0} \rightarrow p \bar{p} K^{* 0}$ and $B^{+} \rightarrow \eta_{c} K^{*+}$ decays and study of the decay dynamics of $B$ meson decays into $p \bar{p} h$ final states, Phys. Rev. D 76, 092004 (2007).

[10] J. H. Chen et al. (Belle Collaboration), Observation of $B^{0} \rightarrow$ $p \bar{p} K^{* 0}$ with a Large $K^{* 0}$ Polarization, Phys. Rev. Lett. 100, 251801 (2008).

[11] C. Patrignani et al. (Particle Data Group Collaboration), Review of particle physics, Chin. Phys. C 40, 100001 (2016).

[12] C. Bebek et al. (CLEO Collaboration), Search for the Charmless Decays $B \rightarrow p \bar{p} \pi$ and $p \bar{p} \pi \pi$, Phys. Rev. Lett. 62, 8 (1989).

[13] A. A. Alves, Jr. et al. (LHCb Collaboration), The LHCb detector at the LHC, J. Instrum. 3, S08005 (2008).

[14] R. Aaij et al. (LHCb Collaboration), LHCb detector performance, Int. J. Mod. Phys. A A30, 1530022 (2015).

[15] T. Sjöstrand, S. Mrenna, and P. Skands, PYTHIA 6.4 physics and manual, J. High Energy Phys. 05 (2006) 026; A brief introduction to PYTHIA 8.1, Comput. Phys. Commun. 178, 852 (2008).

[16] I. Belyaev et al., Handling of the generation of primary events in Gauss, the LHCb simulation framework, J. Phys. Conf. Ser. 331, 032047 (2011).

[17] D. J. Lange, The EvtGen particle decay simulation package, Nucl. Instrum. Methods Phys. Res., Sect. A 462, 152 (2001).

[18] P. Golonka and Z. Was, PHOTOS Monte Carlo: A precision tool for QED corrections in $Z$ and $W$ decays, Eur. Phys. J. C 45, 97 (2006).

[19] J. Allison et al. (Geant4 Collaboration), Geant4 developments and applications, IEEE Trans. Nucl. Sci. 53 (2006) 270; S. Agostinelli et al. (Geant4 Collaboration), Geant4:
A simulation toolkit, Nucl. Instrum. Methods Phys. Res., Sect. A 506, 250 (2003).

[20] M. Clemencic, G. Corti, S. Easo, C. R. Jones, S. Miglioranzi, M. Pappagallo, and P. Robbe, The LHCb simulation application, Gauss: Design, evolution and experience, J. Phys. Conf. Ser. 331, 032023 (2011).

[21] R. Aaij et al., The LHCb trigger and its performance in 2011, J. Instrum. 8, P04022 (2013).

[22] V. V. Gligorov and M. Williams, Efficient, reliable and fast high-level triggering using a bonsai boosted decision tree, J. Instrum. 8, P02013 (2013).

[23] L. Breiman, J. H. Friedman, R. A. Olshen, and C. J. Stone, Classification and Regression Trees (Wadsworth International Group, Belmont, California, USA, 1984).

[24] Y. Freund and R. E. Schapire, A decision-theoretic generalization of on-line learning and an application to boosting, J. Comput. Syst. Sci. 55, 119 (1997).

[25] R. Aaij et al. (LHCb Collaboration), Search for $B_{c}^{+}$decays to the $p \bar{p} \pi^{+}$final state, Phys. Lett. B 759, 313 (2016).

[26] T. Skwarnicki, Ph.D. thesis, Institute of Nuclear Physics, Krakow [Report No. DESY-F31-86-02, 1986].

[27] D. Aston et al., A study of $K^{-} \pi^{+}$scattering in the reaction $K^{-} p \rightarrow K^{-} \pi^{+} n$ at $11 \mathrm{GeV} / \mathrm{c}$, Nucl. Phys. B296, 493 (1988).

[28] B. Aubert et al. (BABAR Collaboration), Time-dependent and time-integrated angular analysis of $B \rightarrow \varphi K_{S}^{0} \pi^{0}$ and $\varphi K^{ \pm} \pi^{\mp}$, Phys. Rev. D 78, 092008 (2008).

[29] S. S. Wilks, The large-sample distribution of the likelihood ratio for testing composite hypotheses, Ann. Math. Stat. 9, 60 (1938).

[30] R. Aaij et al. (LHCb Collaboration), Measurement of the fragmentation fraction ratio $f_{s} / f_{d}$ and its dependence on $B$ meson kinematics, J. High Energy Phys. 04 (2013) 001, $f_{s} / f_{d}$ value updated in LHCb-CONF-2013-011.

[31] M. Adinolfi et al., Performance of the LHCb RICH detector at the LHC, Eur. Phys. J. C 73, 2431 (2013).

[32] L. Anderlini et al., Report No. LHCb-PUB-2016-021.

[33] R. Aaij et al. (LHCb Collaboration), Observation of $B^{0} \rightarrow \bar{D}^{0} K^{+} K^{-}$and Evidence for $B_{s}^{0} \rightarrow \bar{D}^{0} K^{+} K^{-}$, Phys. Rev. Lett. 109, 131801 (2012).

[34] M. Pivk and F. R. Le Diberder, sPlot: A statistical tool to unfold data distributions, Nucl. Instrum. Methods Phys. Res., Sect. A 555, 356 (2005).

[35] K. Chilikin et al. (Belle Collaboration), Observation of a new charged charmoniumlike state in $\bar{B}^{0} \rightarrow J / \psi K^{-} \pi^{+}$ decays, Phys. Rev. D 90, 112009 (2014).

[36] R. Aaij et al. (LHCb Collaboration), Measurement of matter-antimatter differences in beauty baryon decays, Nat. Phys. 13, 391 (2017).

R. Aaij, ${ }^{40}$ B. Adeva, ${ }^{39}$ M. Adinolfi, ${ }^{48}$ Z. Ajaltouni, ${ }^{5}$ S. Akar, ${ }^{59}$ J. Albrecht,${ }^{10}$ F. Alessio, ${ }^{40}$ M. Alexander, ${ }^{53}$ S. Ali, ${ }^{43}$ G. Alkhazov, ${ }^{31}$ P. Alvarez Cartelle, ${ }^{55}$ A. A. Alves Jr., ${ }^{59}$ S. Amato, ${ }^{2}$ S. Amerio, ${ }^{23}$ Y. Amhis, ${ }^{7}$ L. An, ${ }^{3}$ L. Anderlini, ${ }^{18}$ G. Andreassi ${ }^{41}$ M. Andreotti, ${ }^{17, a}$ J. E. Andrews, ${ }^{60}$ R. B. Appleby, ${ }^{56}$ F. Archilli, ${ }^{43}$ P. d'Argent, ${ }^{12}$ J. Arnau Romeu, ${ }^{6}$ A. Artamonov, ${ }^{37}$ M. Artuso, ${ }^{61}$ E. Aslanides, ${ }^{6}$ G. Auriemma, ${ }^{26}$ M. Baalouch, ${ }^{5}$ I. Babuschkin,${ }^{56}$ S. Bachmann, ${ }^{12}$ J. J. Back, ${ }^{50}$ A. Badalov, ${ }^{38}$ C. Baesso, ${ }^{62}$ S. Baker, ${ }^{55}$ V. Balagura, ${ }^{7, b}$ W. Baldini, ${ }^{17}$ A. Baranov,${ }^{35}$ R. J. Barlow,${ }^{56}$ C. Barschel,${ }^{40}$ S. Barsuk, 
W. Barter, ${ }^{56}$ F. Baryshnikov, ${ }^{32}$ M. Baszczyk,${ }^{27, c}$ V. Batozskaya, ${ }^{29}$ B. Batsukh, ${ }^{61}$ V. Battista, ${ }^{41}$ A. Bay, ${ }^{41}$ L. Beaucourt, ${ }^{4}$ J. Beddow, ${ }^{53}$ F. Bedeschi, ${ }^{24}$ I. Bediaga, ${ }^{1}$ A. Beiter, ${ }^{61}$ L. J. Bell, ${ }^{43}$ V. Bellee,${ }^{41}$ N. Belloli, ${ }^{21, d}$ K. Belous,${ }^{37}$ I. Belyaev, ${ }^{32}$ E. Ben-Haim, ${ }^{8}$ G. Bencivenni, ${ }^{19}$ S. Benson, ${ }^{43}$ S. Beranek, ${ }^{9}$ A. Berezhnoy, ${ }^{33}$ R. Bernet, ${ }^{42}$ A. Bertolin, ${ }^{23}$ C. Betancourt, ${ }^{42}$ F. Betti, ${ }^{15}$ M.-O. Bettler, ${ }^{40}$ M. van Beuzekom, ${ }^{43}$ Ia. Bezshyiko, ${ }^{42}$ S. Bifani, ${ }^{47}$ P. Billoir, ${ }^{8}$ A. Birnkraut,${ }^{10}$ A. Bitadze, ${ }^{56}$ A. Bizzeti, ${ }^{18, \mathrm{e}}$ T. Blake, ${ }^{50}$ F. Blanc, ${ }^{41}$ J. Blouw, ${ }^{11}$ S. Blusk,${ }^{61}$ V. Bocci, ${ }^{26}$ T. Boettcher, ${ }^{58}$ A. Bondar, ${ }^{36, f}$ N. Bondar, ${ }^{31}$ W. Bonivento, ${ }^{16}$ I. Bordyuzhin, ${ }^{32}$ A. Borgheresi, ${ }^{21, d}$ S. Borghi, ${ }^{56}$ M. Borisyak, ${ }^{35}$ M. Borsato, ${ }^{39}$ F. Bossu, ${ }^{7}$ M. Boubdir, ${ }^{9}$ T. J. V. Bowcock, ${ }^{54}$ E. Bowen, ${ }^{42}$ C. Bozzi,,${ }^{17,40}$ S. Braun, ${ }^{12}$ T. Britton, ${ }^{61}$ J. Brodzicka, ${ }^{56}$ E. Buchanan, ${ }^{48}$ C. Burr, ${ }^{56}$ A. Bursche, ${ }^{16}$ J. Buytaert, ${ }^{40}$ S. Cadeddu, ${ }^{16}$ R. Calabrese, ${ }^{17, a}$ M. Calvi, ${ }^{21, d}$ M. Calvo Gomez, ${ }^{38, g}$ A. Camboni, ${ }^{38}$ P. Campana, ${ }^{19}$ D. H. Campora Perez, ${ }^{40}$ L. Capriotti,${ }^{56}$ A. Carbone, ${ }^{15, h}$ G. Carboni, ${ }^{25, i}$ R. Cardinale, ${ }^{20, j}$ A. Cardini, ${ }^{16}$ P. Carniti, ${ }^{21, \mathrm{~d}}$ L. Carson ${ }^{52}$ K. Carvalho Akiba, ${ }^{2}$ G. Casse, ${ }^{54}$ L. Cassina, ${ }^{21, d}$ L. Castillo Garcia, ${ }^{41}$ M. Cattaneo, ${ }^{40}$ G. Cavallero, ${ }^{20,40, j}$ R. Cenci, ${ }^{24, \mathrm{k}}$ D. Chamont, ${ }^{7}$ M. Charles, ${ }^{8}$ Ph. Charpentier, ${ }^{40}$ G. Chatzikonstantinidis, ${ }^{47}$ M. Chefdeville, ${ }^{4}$ S. Chen, ${ }^{56}$ S. F. Cheung,${ }^{57}$ V. Chobanova, ${ }^{39}$ M. Chrzaszcz, ${ }^{42,27}$ A. Chubykin, ${ }^{31}$ X. Cid Vidal, ${ }^{39}$ G. Ciezarek,${ }^{43}$ P. E. L. Clarke, ${ }^{52}$ M. Clemencic, ${ }^{40}$ H. V. Cliff, ${ }^{49}$ J. Closier, ${ }^{40}$ V. Coco, ${ }^{59}$ J. Cogan, ${ }^{6}$ E. Cogneras,${ }^{5}$ V. Cogoni, ${ }^{16,1}$ L. Cojocariu, ${ }^{30}$ P. Collins,${ }^{40}$ A. Comerma-Montells, ${ }^{12}$ A. Contu, ${ }^{40}$ A. Cook,${ }^{48}$ G. Coombs,${ }^{40}$ S. Coquereau ${ }^{38}$ G. Corti, ${ }^{40}$ M. Corvo, ${ }^{17, a}$ C. M. Costa Sobral, ${ }^{50}$ B. Couturier, ${ }^{40}$ G. A. Cowan, ${ }^{52}$ D. C. Craik,${ }^{52}$ A. Crocombe, ${ }^{50}$ M. Cruz Torres, ${ }^{62}$ S. Cunliffe, ${ }^{55}$ R. Currie ${ }^{52}$ C. D'Ambrosio, ${ }^{40}$ F. Da Cunha Marinho, ${ }^{2}$ E. Dall'Occo, ${ }^{43}$ J. Dalseno, ${ }^{48}$ A. Davis, ${ }^{3}$ K. De Bruyn, ${ }^{6}$ S. De Capua ${ }^{56}$ M. De Cian, ${ }^{12}$ J. M. De Miranda, ${ }^{1}$ L. De Paula, ${ }^{2}$ M. De Serio, ${ }^{14 m}$ P. De Simone, ${ }^{19}$ C. T. Dean, ${ }^{53}$ D. Decamp, ${ }^{4}$ M. Deckenhoff ${ }^{10}$ L. Del Buono, ${ }^{8}$ H.-P. Dembinski, ${ }^{11}$ M. Demmer, ${ }^{10}$ A. Dendek, ${ }^{28}$ D. Derkach,${ }^{35}$ O. Deschamps, ${ }^{5}$ F. Dettori, ${ }^{54}$ B. Dey, ${ }^{22}$ A. Di Canto,${ }^{40}$ P. Di Nezza, ${ }^{19}$ H. Dijkstra, ${ }^{40}$ F. Dordei,${ }^{40}$ M. Dorigo, ${ }^{41}$ A. Dosil Suárez,${ }^{39}$ A. Dovbnya ${ }^{45}$ K. Dreimanis, ${ }^{54}$ L. Dufour, ${ }^{43}$ G. Dujany, ${ }^{56}$ K. Dungs,${ }^{40}$ P. Durante, ${ }^{40}$ R. Dzhelyadin, ${ }^{37}$ M. Dziewiecki, ${ }^{12}$ A. Dziurda, ${ }^{40}$ A. Dzyuba, ${ }^{31}$ N. Déléage, ${ }^{4}$ S. Easo, ${ }^{51}$ M. Ebert ${ }^{52}$ U. Egede, ${ }^{55}$ V. Egorychev, ${ }^{32}$ S. Eidelman, ${ }^{36, f}$ S. Eisenhardt,${ }^{52}$ U. Eitschberger, ${ }^{10}$ R. Ekelhof, ${ }^{10}$ L. Eklund, ${ }^{53}$ S. Ely, ${ }^{61}$ S. Esen, ${ }^{12}$ H. M. Evans, ${ }^{49}$ T. Evans, ${ }^{57}$ A. Falabella, ${ }^{15}$ N. Farley, ${ }^{47}$ S. Farry, ${ }^{54}$ R. Fay, ${ }^{54}$ D. Fazzini, ${ }^{21, d}$ D. Ferguson, ${ }^{52}$ G. Fernandez,${ }^{38}$ A. Fernandez Prieto, ${ }^{39}$ F. Ferrari, ${ }^{15}$ F. Ferreira Rodrigues, ${ }^{2}$ M. Ferro-Luzzi ${ }^{40}$ S. Filippov,${ }^{34}$ R. A. Fini,${ }^{14}$ M. Fiore, ${ }^{17, a}$ M. Fiorini, ${ }^{17, a}$ M. Firlej,${ }^{28}$ C. Fitzpatrick, ${ }^{41}$ T. Fiutowski, ${ }^{28}$ F. Fleuret, ${ }^{7, n}$ K. Fohl,${ }^{40}$ M. Fontana, ${ }^{16,40}$ F. Fontanelli, ${ }^{20, j}$ D. C. Forshaw, ${ }^{61}$ R. Forty, ${ }^{40}$ V. Franco Lima, ${ }^{54}$ M. Frank, ${ }^{40}$ C. Frei ${ }^{40}$ J. Fu, ${ }^{22,0}$ W. Funk,${ }^{40}$ E. Furfaro, ${ }^{25, \mathrm{i}}$ C. Färber, ${ }^{40}$ A. Gallas Torreira, ${ }^{39}$ D. Galli, ${ }^{15, \mathrm{~h}}$ S. Gallorini, ${ }^{23}$ S. Gambetta, ${ }^{52}$ M. Gandelman, ${ }^{2}$ P. Gandini, ${ }^{57}$ Y. Gao, ${ }^{3}$ L. M. Garcia Martin, ${ }^{69}$ J. García Pardiñas, ${ }^{39}$ J. Garra Tico, ${ }^{49}$

L. Garrido, ${ }^{38}$ P. J. Garsed, ${ }^{49}$ D. Gascon, ${ }^{38}$ C. Gaspar,${ }^{40}$ L. Gavardi, ${ }^{10}$ G. Gazzoni, ${ }^{5}$ D. Gerick, ${ }^{12}$ E. Gersabeck, ${ }^{12}$ M. Gersabeck, ${ }^{56}$ T. Gershon, ${ }^{50} \mathrm{Ph}$. Ghez, ${ }^{4}$ S. Gianì ${ }^{41}$ V. Gibson, ${ }^{49}$ O. G. Girard ${ }^{41}$ L. Giubega,${ }^{30}$ K. Gizdov, ${ }^{52}$ V. V. Gligorov, ${ }^{8}$ D. Golubkov, ${ }^{32}$ A. Golutvin, ${ }^{55,40}$ A. Gomes, ${ }^{1, p}$ I. V. Gorelov, ${ }^{33}$ C. Gotti, ${ }^{21, d}$ E. Govorkova, ${ }^{43}$ R. Graciani Diaz, ${ }^{38}$ L. A. Granado Cardoso, ${ }^{40}$ E. Graugés,${ }^{38}$ E. Graverini, ${ }^{42}$ G. Graziani, ${ }^{18}$ A. Grecu, ${ }^{30}$ R. Greim, ${ }^{9}$ P. Griffith, ${ }^{16}$ L. Grillo, ${ }^{21,40, d}$ B. R. Gruberg Cazon, ${ }^{57}$ O. Grünberg, ${ }^{67}$ E. Gushchin, ${ }^{34}$ Yu. Guz, ${ }^{37}$ T. Gys,${ }^{40}$ C. Göbel, ${ }^{62}$

T. Hadavizadeh ${ }^{57}$ C. Hadjivasiliou, ${ }^{5}$ G. Haefeli, ${ }^{41}$ C. Haen, ${ }^{40}$ S. C. Haines, ${ }^{49}$ B. Hamilton, ${ }^{60}$ X. Han, ${ }^{12}$

S. Hansmann-Menzemer, ${ }^{12}$ N. Harnew ${ }^{57}$ S. T. Harnew, ${ }^{48}$ J. Harrison, ${ }^{56}$ M. Hatch,${ }^{40}$ J. He,${ }^{63}$ T. Head, ${ }^{41}$ A. Heister, ${ }^{9}$

K. Hennessy, ${ }^{54}$ P. Henrard, ${ }^{5}$ L. Henry, ${ }^{69}$ E. van Herwijnen, ${ }^{40}$ M. Heß, ${ }^{67}$ A. Hicheur, ${ }^{2}$ D. Hill,${ }^{57}$ C. Hombach, ${ }^{56}$ P. H. Hopchev, ${ }^{41}$ Z.-C. Huard, ${ }^{59}$ W. Hulsbergen, ${ }^{43}$ T. Humair, ${ }^{55}$ M. Hushchyn, ${ }^{35}$ D. Hutchcroft, ${ }^{54}$ M. Idzik, ${ }^{28}$ P. Ilten, ${ }^{58}$ R. Jacobsson, ${ }^{40}$ J. Jalocha, ${ }^{57}$ E. Jans, ${ }^{43}$ A. Jawahery, ${ }^{60}$ F. Jiang, ${ }^{3}$ M. John, ${ }^{57}$ D. Johnson, ${ }^{40}$ C. R. Jones, ${ }^{49}$ C. Joram, ${ }^{40}$ B. Jost, ${ }^{40}$ N. Jurik, ${ }^{57}$ S. Kandybei, ${ }^{45}$ M. Karacson, ${ }^{40}$ J. M. Kariuki, ${ }^{48}$ S. Karodia, ${ }^{53}$ M. Kecke, ${ }^{12}$ M. Kelsey, ${ }^{61}$ M. Kenzie, ${ }^{49}$ T. Ketel, ${ }^{44}$ E. Khairullin, ${ }^{35}$ B. Khanji, ${ }^{12}$ C. Khurewathanakul, ${ }^{41}$ T. Kirn, ${ }^{9}$ S. Klaver, ${ }^{56}$ K. Klimaszewski, ${ }^{29}$ T. Klimkovich, ${ }^{11}$ S. Koliiev, ${ }^{46}$ M. Kolpin, ${ }^{12}$ I. Komarov ${ }^{41}$ R. Kopecna, ${ }^{12}$ P. Koppenburg, ${ }^{43}$ A. Kosmyntseva, ${ }^{32}$ S. Kotriakhova, ${ }^{31}$ M. Kozeiha, ${ }^{5}$

L. Kravchuk, ${ }^{34}$ M. Kreps,${ }^{50}$ P. Krokovny, ${ }^{36, f}$ F. Kruse, ${ }^{10}$ W. Krzemien, ${ }^{29}$ W. Kucewicz, ${ }^{27, c}$ M. Kucharczyk, ${ }^{27}$

V. Kudryavtsev, ${ }^{36, f}$ A. K. Kuonen, ${ }^{41}$ K. Kurek, ${ }^{29}$ T. Kvaratskheliya, ${ }^{32,40}$ D. Lacarrere, ${ }^{40}$ G. Lafferty, ${ }^{56}$ A. Lai, ${ }^{16}$ G. Lanfranchi, ${ }_{19}$ C. Langenbruch, ${ }^{9}$ T. Latham, ${ }^{50}$ C. Lazzeroni, ${ }^{47}$ R. Le Gac, ${ }^{6}$ J. van Leerdam, ${ }^{43}$ A. Leflat, ${ }^{33,40}$ J. Lefrançois, ${ }^{7}$

R. Lefèvre, ${ }^{5}$ F. Lemaitre, ${ }^{40}$ E. Lemos Cid, ${ }^{39}$ O. Leroy, ${ }^{6}$ T. Lesiak, ${ }^{27}$ B. Leverington, ${ }^{12}$ T. Li ${ }^{3}$ Y. Li, ${ }^{7}$ Z. Li, ${ }^{61}$

T. Likhomanenko, ${ }^{35,68}$ R. Lindner, ${ }^{40}$ F. Lionetto, ${ }^{42}$ X. Liu, ${ }^{3}$ D. Loh ${ }^{50}$ I. Longstaff, ${ }^{53}$ J. H. Lopes, ${ }^{2}$ D. Lucchesi, ${ }^{23, q}$ M. Lucio Martinez, ${ }^{39}$ H. Luo, ${ }^{52}$ A. Lupato, ${ }^{23}$ E. Luppi,,${ }^{17, a}$ O. Lupton, ${ }^{40}$ A. Lusiani, ${ }^{24}$ X. Lyu, ${ }^{63}$ F. Machefert, ${ }^{7}$ F. Maciuc, ${ }^{30}$ O. Maev,${ }^{31}$ K. Maguire,${ }^{56}$ S. Malde,${ }^{57}$ A. Malinin,${ }^{68}$ T. Maltsev,${ }^{36}$ G. Manca,${ }^{16,1}$ G. Mancinelli, ${ }^{6}$ P. Manning, ${ }^{61}$ J. Maratas,,${ }^{5, r}$ J. F. Marchand, ${ }^{4}$ U. Marconi, ${ }^{15}$ C. Marin Benito, ${ }^{38}$ M. Marinangeli, ${ }^{41}$ P. Marino, ${ }^{24, k}$ J. Marks, ${ }^{12}$ G. Martellotti, ${ }^{26}$ M. Martin, ${ }^{6}$ M. Martinelli, ${ }^{41}$ D. Martinez Santos, ${ }^{39}$ F. Martinez Vidal, ${ }^{69}$ D. Martins Tostes, ${ }^{2}$ L. M. Massacrier, ${ }^{7}$ A. Massafferri, ${ }^{1}$ 
R. Matev, ${ }^{40}$ A. Mathad ${ }^{50}$ Z. Mathe ${ }^{40}$ C. Matteuzzi, ${ }^{21}$ A. Mauri, ${ }^{42}$ E. Maurice, ${ }^{7, n}$ B. Maurin, ${ }^{41}$ A. Mazurov, ${ }^{47}$ M. McCann ${ }^{55,40}$ A. McNab,${ }^{56}$ R. McNulty, ${ }^{13}$ B. Meadows ${ }^{59}$ F. Meier, ${ }^{10}$ D. Melnychuk, ${ }^{29}$ M. Merk, ${ }^{43}$ A. Merli, ${ }^{22,40, o}$ E. Michielin, ${ }^{23}$ D. A. Milanes, ${ }^{66}$ M.-N. Minard, ${ }^{4}$ D. S. Mitzel, ${ }^{12}$ A. Mogini, ${ }^{8}$ J. Molina Rodriguez, ${ }^{1}$ I. A. Monroy, ${ }^{66}$ S. Monteil, ${ }^{5}$ M. Morandin, ${ }^{23}$ M. J. Morello, ${ }^{24, k}$ O. Morgunova, ${ }^{68}$ J. Moron, ${ }^{28}$ A. B. Morris,${ }^{52}$ R. Mountain,${ }^{61}$ F. Muheim, ${ }^{52}$ M. Mulder, ${ }^{43}$ M. Mussini, ${ }^{15}$ D. Müller, ${ }^{56}$ J. Müller, ${ }^{10}$ K. Müller, ${ }^{42}$ V. Müller, ${ }^{10}$ P. Naik, ${ }^{48}$ T. Nakada, ${ }^{41}$ R. Nandakumar, ${ }^{51}$ A. Nandi, ${ }^{57}$ I. Nasteva, ${ }^{2}$ M. Needham, ${ }^{52}$ N. Neri, ${ }^{22,40}$ S. Neubert, ${ }^{12}$ N. Neufeld ${ }^{40}$ M. Neuner ${ }^{12}$ T. D. Nguyen, ${ }^{41}$ C. Nguyen-Mau, ${ }^{41, \mathrm{~s}}$ S. Nieswand, ${ }^{9}$ R. Niet,${ }^{10}$ N. Nikitin, ${ }^{33}$ T. Nikodem, ${ }^{12}$ A. Nogay, ${ }^{68}$ A. Novoselov, ${ }^{37}$ D. P. O'Hanlon, ${ }^{50}$ A. Oblakowska-Mucha, ${ }^{28}$ V. Obraztsov, ${ }^{37}$ S. Ogilvy, ${ }^{19}$ R. Oldeman, ${ }^{16,1}$ C. J. G. Onderwater, ${ }^{70}$ A. Ossowska, ${ }^{27}$ J. M. Otalora Goicochea, ${ }^{2}$ P. Owen, ${ }^{42}$ A. Oyanguren, ${ }^{69}$ P. R. Pais, ${ }^{41}$ A. Palano, ${ }^{14 m}$ M. Palutan, ${ }^{19,40}$ A. Papanestis,${ }^{51}$ M. Pappagallo, ${ }^{14, \mathrm{~m}}$ L. L. Pappalardo, ${ }^{17, \mathrm{a}}$ C. Pappenheimer ${ }^{59}$ W. Parker,${ }^{60}$ C. Parkes, ${ }^{56}$ G. Passaleva, ${ }^{18}$ A. Pastore, ${ }^{14, \mathrm{~m}}$ M. Patel,${ }^{55}$ C. Patrignani, ${ }^{15, h}$ A. Pearce, ${ }^{40}$ A. Pellegrino, ${ }^{43}$ G. Penso,${ }^{26}$ M. Pepe Altarelli, ${ }^{40}$ S. Perazzini,${ }^{40}$ P. Perret, ${ }^{5}$ L. Pescatore, ${ }^{41}$ K. Petridis, ${ }^{48}$ A. Petrolini,${ }^{20, j}$ A. Petrov,${ }^{68}$ M. Petruzzo, ${ }^{22,0}$ E. Picatoste Olloqui, ${ }^{38}$ B. Pietrzyk,${ }^{4}$ M. Pikies,${ }^{27}$ D. Pinci, ${ }^{26}$ A. Pistone,${ }^{20, j}$ A. Piucci, ${ }^{12}$ V. Placinta, ${ }^{30}$ S. Playfer, ${ }^{52}$ M. Plo Casasus, ${ }^{39}$ T. Poikela, ${ }^{40}$ F. Polci, ${ }^{8}$ M. Poli Lener, ${ }^{19}$ A. Poluektov, ${ }^{50,36}$ I. Polyakov, ${ }^{61}$ E. Polycarpo, ${ }^{2}$ G. J. Pomery, ${ }^{48}$ S. Ponce,${ }^{40}$ A. Popov, ${ }^{37}$ D. Popov,${ }^{11,40}$ B. Popovici, ${ }^{30}$ S. Poslavskii, ${ }^{37}$ C. Potterat, ${ }^{2}$ E. Price, ${ }^{48}$ J. Prisciandaro, ${ }^{39}$ C. Prouve,${ }^{48}$ V. Pugatch,${ }^{46}$ A. Puig Navarro, ${ }^{42}$ G. Punzi, ${ }^{24, t}$ C. Qian, ${ }^{63}$ W. Qian, ${ }^{50}$ R. Quagliani, ${ }^{7,48}$ B. Rachwal, ${ }^{28}$ J. H. Rademacker, ${ }^{48}$ M. Rama, ${ }^{24}$ M. Ramos Pernas,${ }^{39}$ M. S. Rangel, ${ }^{2}$ I. Raniuk, ${ }^{45}$ F. Ratnikov, ${ }^{35}$ G. Raven, ${ }^{44}$ F. Redi,${ }^{55}$ S. Reichert, ${ }^{10}$ A. C. dos Reis, ${ }^{1}$ C. Remon Alepuz ${ }^{69}$ V. Renaudin, ${ }^{7}$ S. Ricciardi, ${ }^{51}$ S. Richards, ${ }^{48}$ M. Rihl,${ }^{40}$ K. Rinnert, ${ }^{54}$ V. Rives Molina, ${ }^{38}$ P. Robbe, ${ }^{7}$ A. B. Rodrigues, ${ }^{1}$ E. Rodrigues, ${ }^{59}$ J. A. Rodriguez Lopez, ${ }^{66}$ P. Rodriguez Perez,${ }^{56}$ A. Rogozhnikov, ${ }^{35}$ S. Roiser ${ }^{40}$ A. Rollings, ${ }^{57}$ V. Romanovskiy, ${ }^{37}$ A. Romero Vidal, ${ }^{39}$ J. W. Ronayne, ${ }^{13}$ M. Rotondo, ${ }^{19}$ M. S. Rudolph, ${ }^{61}$ T. Ruf, ${ }^{40}$ P. Ruiz Valls, ${ }^{69}$ J. J. Saborido Silva, ${ }^{39}$ E. Sadykhov, ${ }^{32}$ N. Sagidova, ${ }^{31}$ B. Saitta, ${ }^{16,1}$ V. Salustino Guimaraes, ${ }^{1}$ D. Sanchez Gonzalo, ${ }^{38}$ C. Sanchez Mayordomo, ${ }^{69}$ B. Sanmartin Sedes, ${ }^{39}$ R. Santacesaria, ${ }^{26}$ C. Santamarina Rios, ${ }^{39}$ M. Santimaria, ${ }^{19}$ E. Santovetti, ${ }^{25, i}$ A. Sarti, ${ }^{19, u}$ C. Satriano, ${ }^{26, v}$ A. Satta, ${ }^{25}$ D. M. Saunders, ${ }^{48}$ D. Savrina, ${ }^{32,33}$ S. Schael, ${ }^{9}$ M. Schellenberg, ${ }^{10}$ M. Schiller, ${ }^{53}$ H. Schindler, ${ }^{40}$ M. Schlupp, ${ }^{10}$ M. Schmelling, ${ }^{11}$ T. Schmelzer, ${ }^{10}$ B. Schmidt, ${ }^{40}$ O. Schneider, ${ }^{41}$ A. Schopper, ${ }^{40}$ H. F. Schreiner,${ }^{59}$ K. Schubert, ${ }^{10}$ M. Schubiger, ${ }^{41}$ M.-H. Schune, ${ }^{7}$ R. Schwemmer, ${ }^{40}$ B. Sciascia, ${ }^{19}$ A. Sciubba, ${ }^{26, u}$ A. Semennikov, ${ }^{32}$ A. Sergi, ${ }^{47}$ N. Serra, ${ }^{42}$ J. Serrano, ${ }^{6}$ L. Sestini, ${ }^{23}$ P. Seyfert, ${ }^{21}$ M. Shapkin, ${ }^{37}$ I. Shapoval, ${ }^{45}$ Y. Shcheglov, ${ }^{31}$ T. Shears,${ }^{54}$ L. Shekhtman, ${ }^{36, f}$ V. Shevchenko, ${ }^{68}$ B. G. Siddi, ${ }^{17,40}$ R. Silva Coutinho, ${ }^{42}$ L. Silva de Oliveira, ${ }^{2}$ G. Simi,${ }^{23, q}$ S. Simone, ${ }^{14, m}$ M. Sirendi, ${ }^{49}$ N. Skidmore, ${ }^{48}$ T. Skwarnicki, ${ }^{61}$ E. Smith ${ }^{55}$ I. T. Smith ${ }^{52}$ J. Smith, ${ }^{49}$ M. Smith, ${ }^{55}$ 1. Soares Lavra, ${ }^{1}$ M. D. Sokoloff, ${ }^{59}$ F. J. P. Soler, ${ }^{53}$ B. Souza De Paula, ${ }^{2}$ B. Spaan, ${ }^{10}$ P. Spradlin, ${ }^{53}$ S. Sridharan, ${ }^{40}$ F. Stagni, ${ }^{40}$ M. Stahl, ${ }^{12}$ S. Stahl,${ }^{40}$ P. Stefko, ${ }^{41}$ S. Stefkova, ${ }^{55}$ O. Steinkamp, ${ }^{42}$ S. Stemmle, ${ }^{12}$ O. Stenyakin, ${ }^{37}$ H. Stevens, ${ }^{10}$ S. Stoica, ${ }^{30}$ S. Stone,${ }^{61}$ B. Storaci, ${ }^{42}$ S. Stracka, ${ }^{24, t}$ M. E. Stramaglia, ${ }^{41}$ M. Straticiuc, ${ }^{30}$ U. Straumann, ${ }^{42}$ L. Sun,${ }^{64}$ W. Sutcliffe,${ }^{55}$ K. Swientek, ${ }^{28}$ V. Syropoulos, ${ }^{44}$ M. Szczekowski, ${ }^{29}$ T. Szumlak, ${ }^{28}$ S. T'Jampens, ${ }^{4}$ A. Tayduganov, ${ }^{6}$ T. Tekampe, ${ }^{10}$ G. Tellarini, ${ }^{17, a}$ F. Teubert, ${ }^{40}$ E. Thomas, ${ }^{40}$ J. van Tilburg, ${ }^{43}$ M. J. Tilley ${ }^{55}$ V. Tisserand, ${ }^{4}$ M. Tobin, ${ }^{41}$ S. Tolk, ${ }^{49}$ L. Tomassetti, ${ }^{17, a}$ D. Tonelli, ${ }^{24}$ S. Topp-Joergensen, ${ }^{57}$ F. Toriello, ${ }^{61}$ R. Tourinho Jadallah Aoude, ${ }^{1}$ E. Tournefier, ${ }^{4}$ S. Tourneur, ${ }^{41} \mathrm{~K}$. Trabelsi, ${ }^{41}$ M. Traill, ${ }^{53}$ M. T. Tran, ${ }^{41}$ M. Tresch, ${ }^{42}$ A. Trisovic, ${ }^{40}$ A. Tsaregorodtsev, ${ }^{6}$ P. Tsopelas, ${ }^{43}$ A. Tully, ${ }^{49}$ N. Tuning, ${ }^{43}$ A. Ukleja, ${ }^{29}$ A. Ustyuzhanin, ${ }^{35}$ U. Uwer, ${ }^{12}$ C. Vacca, ${ }^{16,1}$ V. Vagnoni, ${ }^{15,40}$ A. Valassi, ${ }^{40}$ S. Valat, ${ }^{40}$ G. Valenti, ${ }^{15}$ R. Vazquez Gomez, ${ }^{19}$ P. Vazquez Regueiro, ${ }^{39}$ S. Vecchi, ${ }^{17}$ M. van Veghel,,${ }^{43}$ J. J. Velthuis, ${ }^{48}$ M. Veltri, ${ }^{18, w}$ G. Veneziano, ${ }^{57}$ A. Venkateswaran ${ }^{61}$ T. A. Verlage, ${ }^{9}$ M. Vernet, ${ }^{5}$ M. Vesterinen, ${ }^{12}$ J. V. Viana Barbosa ${ }^{40}$ B. Viaud ${ }^{7}$ D. Vieira,${ }^{63}$ M. Vieites Diaz, ${ }^{39}$ H. Viemann, ${ }^{67}$ X. Vilasis-Cardona, ${ }^{38, g}$ M. Vitti, ${ }^{49}$ V. Volkov, ${ }^{33}$ A. Vollhardt, ${ }^{42}$ B. Voneki, ${ }^{40}$ A. Vorobyev, ${ }^{31}$ V. Vorobyev, ${ }^{36, f}$ C. Voß, ${ }^{9}$ J. A. de Vries ${ }^{43}$ C. Vázquez Sierra ${ }^{39}$ R. Waldi, ${ }^{67}$ C. Wallace,${ }^{50}$ R. Wallace ${ }^{13}$ J. Walsh, ${ }^{24}$ J. Wang, ${ }^{61}$ D. R. Ward, ${ }^{49}$ H. M. Wark, ${ }^{54}$ N. K. Watson, ${ }^{47}$ D. Websdale, ${ }^{55}$ A. Weiden, ${ }^{42}$ M. Whitehead, ${ }^{40}$ J. Wicht,${ }^{50}$ G. Wilkinson, ${ }^{57,40}$ M. Wilkinson, ${ }^{61}$ M. Williams, ${ }^{40}$ M. P. Williams, ${ }^{47}$ M. Williams, ${ }^{58}$ T. Williams, ${ }^{47}$ F. F. Wilson, ${ }^{51}$ J. Wimberley ${ }_{5}^{60}$ M. A. Winn, ${ }^{7}$ J. Wishahi, ${ }^{10}$ W. Wislicki, ${ }^{29}$ M. Witek, ${ }^{27}$ G. Wormser, ${ }^{7}$ S. A. Wotton, ${ }^{49}$ K. Wraight,${ }^{53}$ K. Wyllie, ${ }^{40}$ Y. Xie, ${ }^{65}$ Z. Xu, ${ }^{4}$ Z. Yang, ${ }^{3}$ Z. Yang, ${ }^{60}$ Y. Yao, ${ }^{61}$ H. Yin, ${ }^{65}$ J. Yu, ${ }^{65}$ X. Yuan, ${ }^{36, f}$ O. Yushchenko, ${ }^{37}$ K. A. Zarebski, ${ }^{47}$ M. Zavertyaev, ${ }^{11, b}$ L. Zhang, ${ }^{3}$ Y. Zhang, ${ }^{7}$ A. Zhelezov, ${ }^{12}$ Y. Zheng, ${ }^{63}$ X. Zhu, ${ }^{3}$ V. Zhukov, ${ }^{33}$ and S. Zucchelli ${ }^{15}$

(LHCb Collaboration) 
${ }^{1}$ Centro Brasileiro de Pesquisas Físicas $(C B P F)$, Rio de Janeiro, Brazil

${ }^{2}$ Universidade Federal do Rio de Janeiro (UFRJ), Rio de Janeiro, Brazil

${ }^{3}$ Center for High Energy Physics, Tsinghua University, Beijing, China

${ }^{4} L A P P$, Université Savoie Mont-Blanc, CNRS/IN2P3, Annecy-Le-Vieux, France

${ }^{5}$ Clermont Université, Université Blaise Pascal, CNRS/IN2P3, LPC, Clermont-Ferrand, France

${ }^{6}$ CPPM, Aix-Marseille Université, CNRS/IN2P3, Marseille, France

${ }^{7}$ LAL, Université Paris-Sud, CNRS/IN2P3, Orsay, France

${ }^{8}$ LPNHE, Université Pierre et Marie Curie, Université Paris Diderot, CNRS/IN2P3, Paris, France

${ }^{9}$ I. Physikalisches Institut, RWTH Aachen University, Aachen, Germany

${ }^{10}$ Fakultät Physik, Technische Universität Dortmund, Dortmund, Germany

${ }^{11}$ Max-Planck-Institut für Kernphysik (MPIK), Heidelberg, Germany

${ }^{12}$ Physikalisches Institut, Ruprecht-Karls-Universität Heidelberg, Heidelberg, Germany

${ }^{13}$ School of Physics, University College Dublin, Dublin, Ireland

${ }^{14}$ Sezione INFN di Bari, Bari, Italy

${ }^{15}$ Sezione INFN di Bologna, Bologna, Italy

${ }^{16}$ Sezione INFN di Cagliari, Cagliari, Italy

${ }^{17}$ Universita e INFN, Ferrara, Ferrara, Italy

${ }^{18}$ Sezione INFN di Firenze, Firenze, Italy

${ }^{19}$ Laboratori Nazionali dell'INFN di Frascati, Frascati, Italy

${ }^{20}$ Sezione INFN di Genova, Genova, Italy

${ }^{21}$ Universita \& INFN, Milano-Bicocca, Milano, Italy

${ }^{22}$ Sezione di Milano, Milano, Italy

${ }^{23}$ Sezione INFN di Padova, Padova, Italy

${ }^{24}$ Sezione INFN di Pisa, Pisa, Italy

${ }^{25}$ Sezione INFN di Roma Tor Vergata, Roma, Italy

${ }^{26}$ Sezione INFN di Roma La Sapienza, Roma, Italy

${ }^{27}$ Henryk Niewodniczanski Institute of Nuclear Physics Polish Academy of Sciences, Kraków, Poland

${ }^{28}$ AGH - University of Science and Technology, Faculty of Physics and Applied Computer Science, Kraków, Poland

${ }^{29}$ National Center for Nuclear Research (NCBJ), Warsaw, Poland

${ }^{30}$ Horia Hulubei National Institute of Physics and Nuclear Engineering, Bucharest-Magurele, Romania

${ }^{31}$ Petersburg Nuclear Physics Institute (PNPI), Gatchina, Russia

${ }^{32}$ Institute of Theoretical and Experimental Physics (ITEP), Moscow, Russia

${ }^{33}$ Institute of Nuclear Physics, Moscow State University (SINP MSU), Moscow, Russia

${ }^{34}$ Institute for Nuclear Research of the Russian Academy of Sciences (INR RAN), Moscow, Russia

${ }^{35}$ Yandex School of Data Analysis, Moscow, Russia

${ }^{36}$ Budker Institute of Nuclear Physics (SB RAS), Novosibirsk, Russia

${ }^{37}$ Institute for High Energy Physics (IHEP), Protvino, Russia

${ }^{38}$ ICCUB, Universitat de Barcelona, Barcelona, Spain

${ }^{39}$ Universidad de Santiago de Compostela, Santiago de Compostela, Spain

${ }^{40}$ European Organization for Nuclear Research (CERN), Geneva, Switzerland

${ }^{41}$ Institute of Physics, Ecole Polytechnique Fédérale de Lausanne (EPFL), Lausanne, Switzerland

${ }^{42}$ Physik-Institut, Universität Zürich, Zürich, Switzerland

${ }^{43}$ Nikhef National Institute for Subatomic Physics, Amsterdam, Netherlands

${ }^{44}$ Nikhef National Institute for Subatomic Physics and VU University Amsterdam, Amsterdam, Netherlands

${ }^{45}$ NSC Kharkiv Institute of Physics and Technology (NSC KIPT), Kharkiv, Ukraine

${ }^{46}$ Institute for Nuclear Research of the National Academy of Sciences (KINR), Kyiv, Ukraine

${ }^{47}$ University of Birmingham, Birmingham, United Kingdom

${ }^{48}$ H.H. Wills Physics Laboratory, University of Bristol, Bristol, United Kingdom

${ }^{49}$ Cavendish Laboratory, University of Cambridge, Cambridge, United Kingdom

${ }^{50}$ Department of Physics, University of Warwick, Coventry, United Kingdom

${ }^{51}$ STFC Rutherford Appleton Laboratory, Didcot, United Kingdom

${ }^{52}$ School of Physics and Astronomy, University of Edinburgh, Edinburgh, United Kingdom

${ }^{53}$ School of Physics and Astronomy, University of Glasgow, Glasgow, United Kingdom

${ }^{54}$ Oliver Lodge Laboratory, University of Liverpool, Liverpool, United Kingdom

${ }^{55}$ Imperial College London, London, United Kingdom

${ }^{56}$ School of Physics and Astronomy, University of Manchester, Manchester, United Kingdom

${ }^{57}$ Department of Physics, University of Oxford, Oxford, United Kingdom

${ }^{58}$ Massachusetts Institute of Technology, Cambridge, Massachusetts, USA

${ }^{59}$ University of Cincinnati, Cincinnati, Ohio, USA 


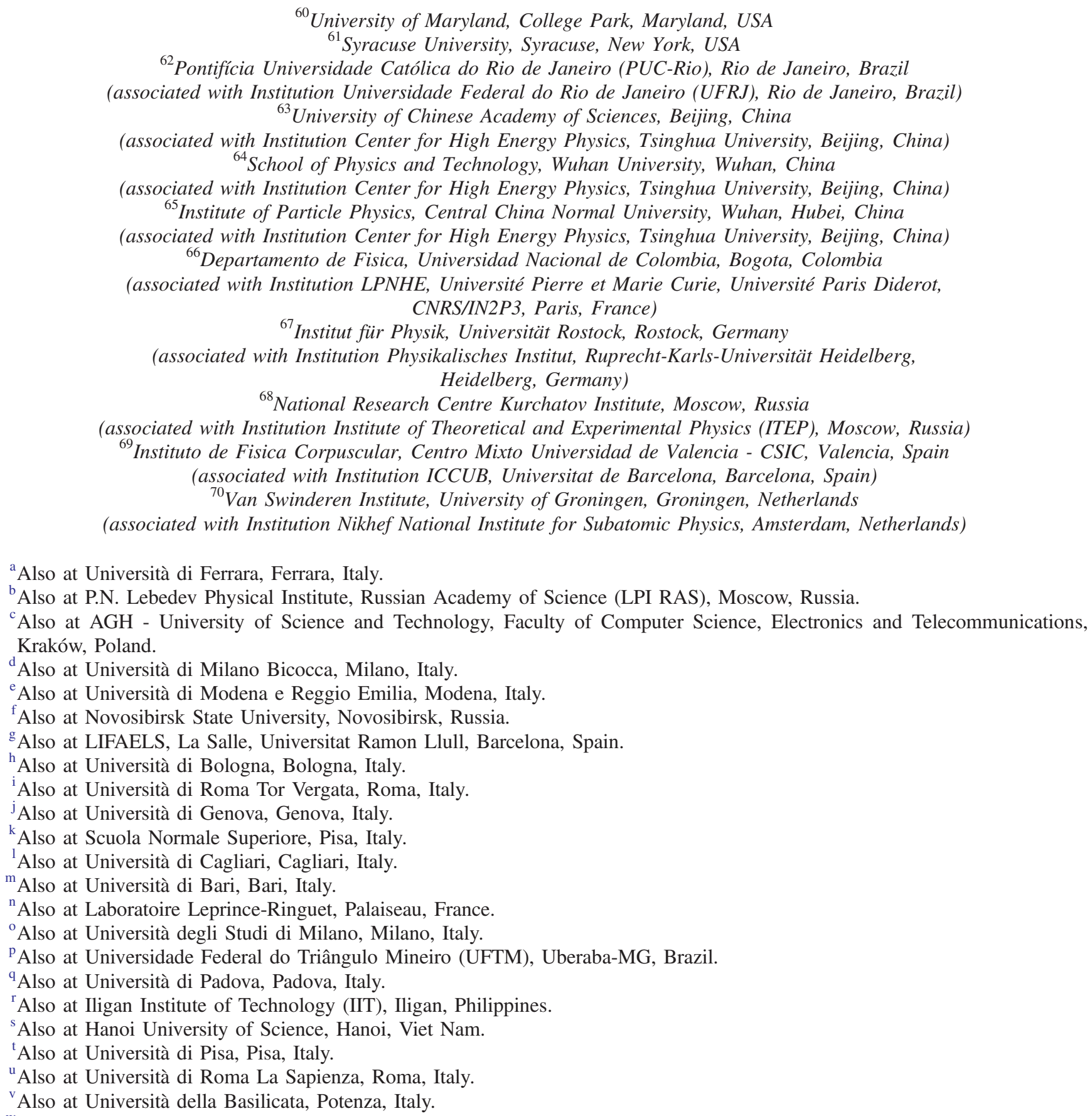

${ }^{\mathrm{b}}$ Also at P.N. Lebedev Physical Institute, Russian Academy of Science (LPI RAS), Moscow, Russia.

${ }^{c}$ Also at AGH - University of Science and Technology, Faculty of Computer Science, Electronics and Telecommunications,

${ }^{\mathrm{d}}$ Also at Università di Milano Bicocca, Milano, Italy.

${ }^{\mathrm{e}}$ Also at Università di Modena e Reggio Emilia, Modena, Italy.

${ }^{\mathrm{f}}$ Also at Novosibirsk State University, Novosibirsk, Russia.

${ }^{g}$ Also at LIFAELS, La Salle, Universitat Ramon Llull, Barcelona, Spain.

${ }^{\mathrm{h}}$ Also at Università di Bologna, Bologna, Italy.

${ }^{\mathrm{i}}$ Also at Università di Roma Tor Vergata, Roma, Italy.

${ }^{\mathrm{k}}$ Also at Scuola Normale Superiore, Pisa, Italy.

${ }^{1}$ Also at Università di Cagliari, Cagliari, Italy.

${ }^{\mathrm{n}}$ Also at Laboratoire Leprince-Ringuet, Palaiseau, France.

${ }^{\circ}$ Also at Università degli Studi di Milano, Milano, Italy.

${ }^{\mathrm{p}}$ Also at Universidade Federal do Triângulo Mineiro (UFTM), Uberaba-MG, Brazil.

${ }^{\mathrm{r}}$ Also at Iligan Institute of Technology (IIT), Iligan, Philippines.

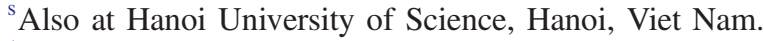

uAlso at Università di Roma La Sapienza, Roma, Italy.

${ }^{v}$ Also at Università della Basilicata, Potenza, Italy.

${ }^{a}$ Also at Università di Ferrara, Ferrara, Italy. Kraków, Poland.

${ }^{\mathrm{j}}$ Also at Università di Genova, Genova, Italy.

${ }^{\mathrm{m}}$ Also at Università di Bari, Bari, Italy.

${ }^{\mathrm{q}}$ Also at Università di Padova, Padova, Italy.

${ }^{\mathrm{t}}$ Also at Università di Pisa, Pisa, Italy.

${ }^{\mathrm{w}}$ Also at Università di Urbino, Urbino, Italy. 